\title{
Compact Hash Tables for Decision-trees ${ }^{\text {th }}$
}

\author{
Yun R. Qu, Viktor K. Prasanna \\ Ming Hsieh Department of Electrical Engineering \\ University of Southern California, Los Angeles, CA 90089 \\ \{yunqu,prasanna\}@usc.edu
}

\begin{abstract}
Decision-trees have been widely used in scientific computing. It is challenging to sustain high performance for large decision-trees. In this paper, we present a conversion technique translating a generic decision-tree into multiple compact hash tables; the conversion technique does not depend on the depth or shape of the decision-tree. All the compact hash tables are searched individually; the outcomes from all the tables are merged into the final result. To evaluate the performance, we prototype our design on state-of-the-art FPGA and multi-core General Purpose Processors (GPPs). Experimental results show that, for a typical 92-leaf decision-tree, we achieve 533 Million Classifications Per Second (MCPS) throughput and 26 ns latency on FPGA, and 134 MCPS throughput and 239 ns latency on multi-core GPP. We sustain $6 \times$ and $2.7 \times$ speedups, respectively.
\end{abstract}

Keywords: decision-tree, hash table, performance, FPGA, multi-core

\section{Introduction}

Tree structures [1] have been widely used for high-performance computation; a tree can be used as the underlying data structure for search or lookup operations. In particular, binary decision-tree has been widely studied due to its simple structure; elegant algorithms based on balanced BST have been proposed [2] for efficient searching of input data.

\footnotetext{
"Supported by the U.S. National Science Foundation under grants CCF-1320211 and ACI-1339756. Equipment grant from Xilinx is gratefully acknowledged.
}

Preprint submitted to Parallel Computing

August 5, 2015

(C) 2015. This manuscript version is made available under the Elsevier user license

http://www.elsevier.com/open-access/userlicense/1.0/ 
State-of-the-art Field Programmable Gate Arrays (FPGAs) [3], with their flexibility and reconfigurability, are especially suitable for implementing a large tree structure [4]. Many hardware architectures have been proposed to enhance the performance for balanced tree structures [5], but it is still challenging to sustain high throughput for large trees on FPGA.

Using software accelerators and virtual machines is a new trend in parallel computing $[6,7]$. State-of-the-art GPP [8] is an attractive platform for low-latency applications. Efficient algorithms are still needed to explore the parallelism and improve the performance of the multi-core processors [9-11].

In this paper, we present a conversion from the decision-trees to a set of compact hash tables for efficient implementation. We map this structure onto various platforms. Specifically, our contributions include the following:

- We provide the conversion technique from trees to compact hash tables; this technique does not depend on the depth or shape of a given decision-tree.

- We evaluate the performance on various state-of-the-art platforms including FPGA and multi-core GPP. We present a thorough performance comparison between these platforms.

- We sustain 533 MCPS throughput (26 ns latency) and 134 MCPS throughput (239 ns latency) for a typical decision-tree used for Internet traffic classification on FPGA and multi-core GPP, respectively. The corresponding speedups on these platforms are $6 \times$ and $2.7 \times$, respectively.

The rest of the paper is organized as follows: Section 2 covers the related work. We introduce the relevant data structures and algorithms in Section 3. Section 4 evaluates the performance. Section 5 concludes this paper.

\section{Background}

\subsection{Generic Decision-tree}

A feature is a distinct set of data (e.g., Internet packet size, date, time, etc.). A generic decision-tree (not necessarily balanced) consists of $N$ leaves. Each internal node specifies a criterion defined on a specific feature. The input data are checked against the criterion of an internal node; the comparison result determines the next-hop, until a leaf node has been reached. We 


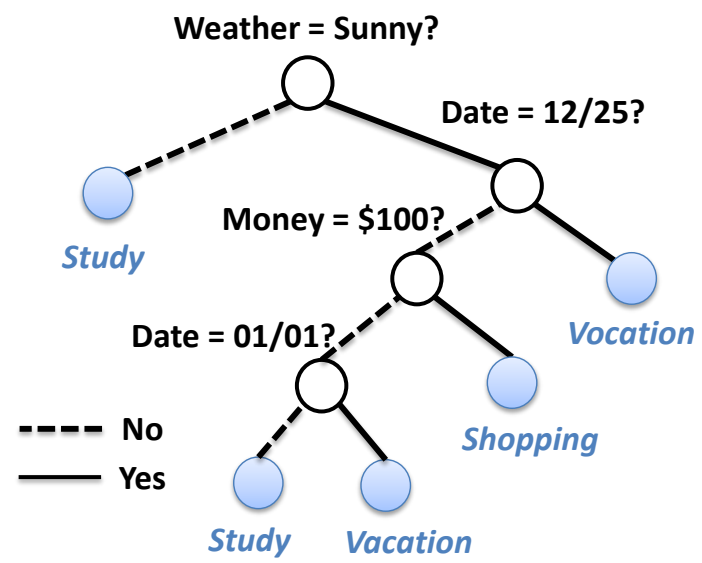

Figure 1: An example of a (static) decision-tree $T$

denote this tree traversal process as classification. The classification process stops at a leaf node, where a final decision can be made.

We show a toy example of a binary decision-tree used for holiday plans in Figure 1. In this example, the decision-tree are built on 3 features: weather, date, and money. Without loss of generality, in this paper, we always assume a left child is to be searched next if the criteria of a node is not satisfied (following a dashed "No"-edge in Figure 1); otherwise a right child is to be searched (following a solid "Yes"-edge). A feature can be used more than once along a path from the root to a leaf. We use $M$ to denote the number of features examined by a decision-tree.

The design of a dynamic decision-tree [12] based on dynamic data is also an interesting problem. However, in this paper, we assume we are given an arbitrary static decision-tree, hence the discussion on dynamic updates of a decision-tree is beyond the scope of this paper.

\subsection{Implementations for Trees}

A lot of the existing works have used pipelining techniques to search binary trees on FPGA $[5,13]$. In a pipelined implementation, each tree level is mapped into an individual pipeline stage; each stage consists of a logic component and a memory module. We denote this implementation $[5,13]$ as the state-of-the-art implementation on FPGA. Denoting $\Omega$ as the total number of nodes in a tree, the state-of-the-art implementation of a decisiontree is expensive: A tree (possibly imbalanced) may require $O(\Omega)$ pipeline 
Table 1: Notations for converting the decision-tree to hash tables

\begin{tabular}{|c|c|}
\hline $\begin{array}{c}A_{n}=\left(S_{0}, S_{1}, \ldots, S_{k_{n}}, \ldots, S_{K_{n}-1}\right), \\
n=0,1, \ldots, N-1, k_{n}=0,1, \ldots, K_{n}-1\end{array}$ & $\begin{array}{c}N \text { root-to-leaf paths in } T, \\
\text { each path visiting } K_{n} \text { edges }\end{array}$ \\
\hline $\operatorname{par}\left\{S_{k_{n}}\right\}, \operatorname{chi}\left\{S_{k_{n}}\right\}$ & parent and child nodes connecting edge $S_{k_{n}}$ \\
\hline$u_{i}^{(m)}, m=0,1, \ldots, M-1$, & $\begin{array}{c}\text { Unique values for } M \text { features, } \\
q^{(m)} \text { unique values for feature } m\end{array}$ \\
\hline$i=0,1, \ldots, q^{(m)}-1$ & $q^{(m)}$ BVs for feature $m$, \\
\hline$B_{i}^{(m)}=\overline{b_{i, 0}^{(m)} b_{i, 1}^{(m)} \ldots b_{i, n}^{(m)} \ldots b_{i, N-1}^{(m)}}$, & each BV consisting of $N$ bits \\
\hline$i=0,1, \ldots, q^{(m)}-1, n=0,1, \ldots, N-1$ & $P$ hash functions for feature $m$ \\
\hline$f_{0}^{(m)}, f_{1}^{(m)}, \ldots, f_{p}^{(m)}, \ldots, f_{P-1}^{(m)}$ & Hash table for feature $m$ \\
\hline$H^{(m)}$ & A set of data structures with different $m$ \\
\hline$[\cdot]_{m}$ &
\end{tabular}

stages, while a lookup may take $O(\Omega)$ time. Besides FPGA, a thorough evaluation for decision-trees on state-of-the-art multi-core GPP is still to be studied.

\section{Data Structures \& Algorithms}

\subsection{Overview}

Our work is inspired by the following observation on a decision-tree $T$ : The classification process on $T$ is a serial process incurring large latency. To reduce the processing latency, more parallelism has to be explored on $T$. In this paper, we examine all the $M$ input features in parallel to reduce the processing latency. In addition, efficient hashing techniques [14] are exploited to examine each input feature. The searching outcomes for all the $M$ inputs can be merged together to produce the final classification result. This final classification result indicates what the final decision should be if we use the $M$ input features to search $T$.

\subsection{Example: A Decision-tree used for Internet Traffic Classification}

Before we present our algorithm, we revisit Bit Vector (BV) [14], a data structure used to efficiently merge the partial results. A BV consists of $N$ bits; the $n$-th bit $(n=0,1, \ldots, N-1)$ is set to " 1 " if and only if the input matches or satisfies the $n$-th condition. Note the number of leaf nodes $(N)$ is 


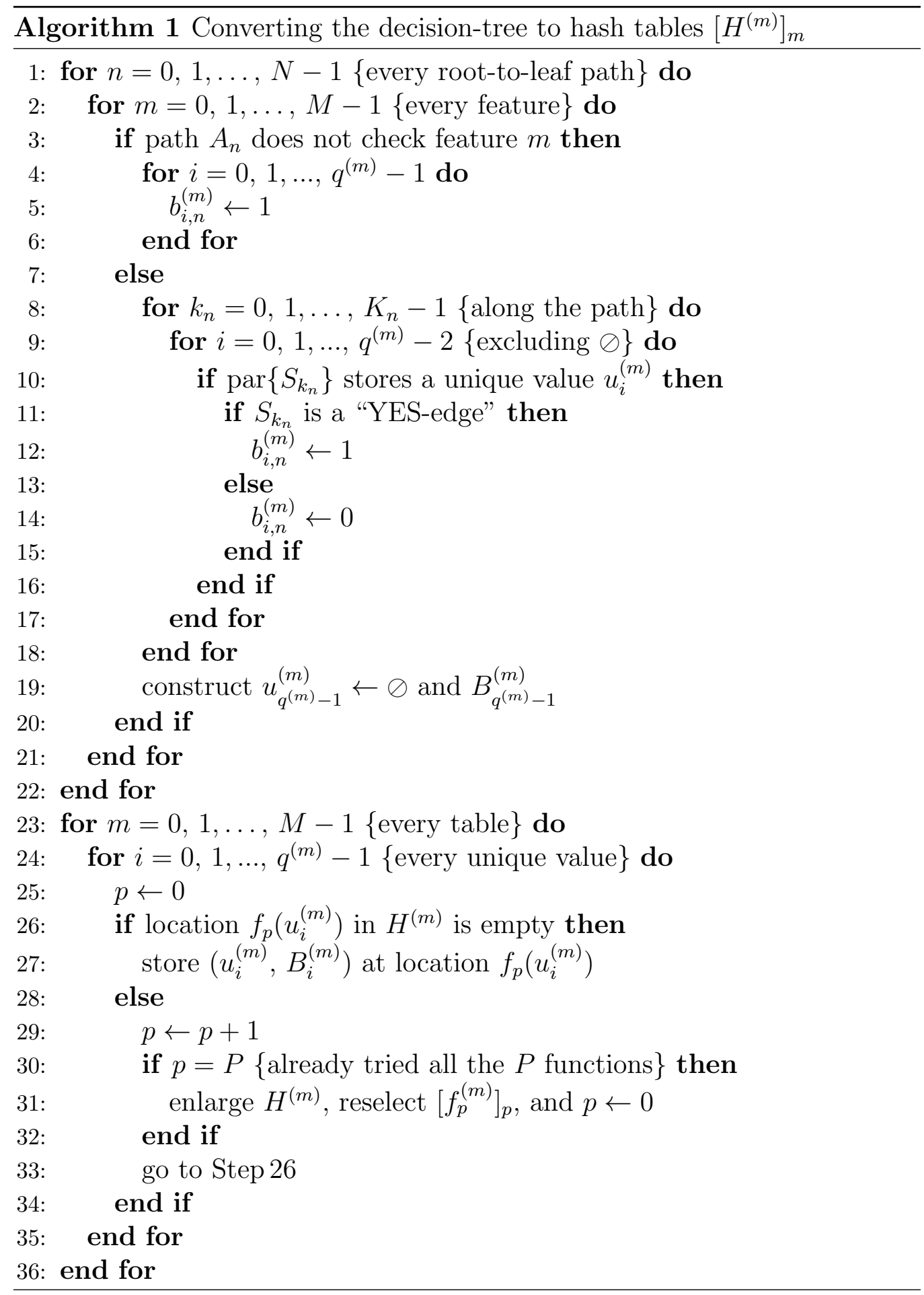



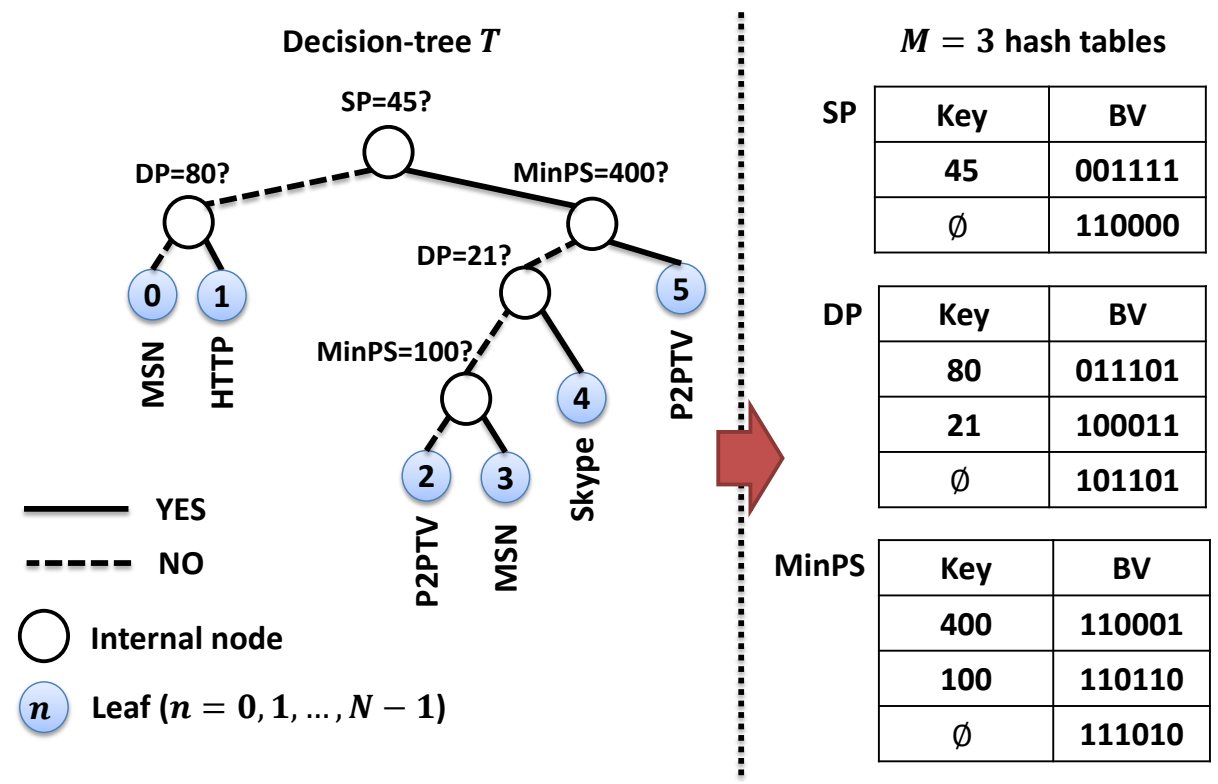

Figure 2: Translating a decision-tree $T$ into $M$ hash tables

usually small in $T$; merging a set of short BVs can be very fast. This is the reason why we choose to use $\mathrm{BV}$ to represent the partial searching results.

During the training phase, we convert $T$ into $M$ hash tables, each constructed for a specific feature. Using the notations in Table 1, we show the pseudo-code in Algorithm 1. After the conversion, each hash table stores a number of $q^{(m)}$ entries, each consisting of a unique value and a BV. A detailed explanation can be found in [14].

We show an example of translating a decision-tree $T$ into $M$ hash tables in Figure 2, where $T$ is used for Internet traffic classification. 3 features are used in this example, including Source Port Number (SP), Destination Port number (DP), and Minimum Packet Size (MinPS).

In Figure 2, 3 compact hash tables are constructed, each consisting of $q^{(m)}$ key-BV pairs $\left(m=0,1,2\right.$, and $q^{(m)}=2,3,3$, respectively). In a specific key-BV pair, a key corresponds to a feature value tested in $T$ (e.g., for feature $\mathrm{SP}, 45$ is the value tested in the root node of $T$ ). A BV has $N$ bits, each bit corresponding to a leaf node of $T$; a bit is set to "1" only if the classification process based on the key can lead to the corresponding leaf node. Note each hash table also stores a " $\oslash$ " as a key, in case the input feature does not match any value tested in $T$. 


\subsection{Searching and Merging}

After the compact hash tables are constructed, the classification process is straightforward. Perfect hashing [15] is employed to search each feature, each returning an $N$-bit BV. All the $M$ BVs are merged by bitwise AND operations to produce the final classification result. To continue the example in Figure 2, let us suppose the input features are: $\mathrm{SP}=45, \mathrm{DP}=125$, and MinPS $=100$. The $3 \mathrm{BVs}$ extracted from the compact hash tables are "001111", "101101", and "110110", respectively. After bitwise ANDing, the final classification result is " 000100 ", indicating the final decision on the Internet traffic class is "MSN" (leaf node 3). Detailed space/time complexity analysis can be found in [14].

\section{Performance Evaluation}

Although the conversion technique leads to the same data structures on FPGA and on multi-core GPP, we use different implementations to achieve as high performance as possible on the two platforms ${ }^{1}$ :

- On FPGA, we customize the pipeline architecture to sustain high clock rate (pipelined implementation).

- On Multi-core GPP, we design parallel algorithms to achieve high performance (parallel implementation).

\subsection{Experimental Setup}

\subsubsection{FPGA-based Implementation}

We conducted experiments using Verilog on Xilinx Vivado Design Suite 2013.4, targeting Virtex-7 XC7VX1140t FLG1930 -2L FPGA [3]. This device has $1100 \mathrm{I} / \mathrm{O}$ pins, 218800 logic slices, $67 \mathrm{Mb}$ BRAM, and can be configured to realize large amount of distributed RAM (distRAM, up to $17 \mathrm{Mb}$ ). Maximum achievable clock rate and resource consumption are shown using post-placeand-route timing reports and resource utilization reports, respectively.

The architecture of our FPGA-based implementations is shown in Figure 3. Based on the $m$-th input feature of width $W_{m}(m=0,1, \ldots, M-1)$, the $m$-th Processing Element $(\mathrm{PE})$ is responsible for extracting a BV from a

\footnotetext{
${ }^{1}$ FPGA integrates reconfigurable logic with fast on-chip memory, while multi-core GPP consists of many parallel processor cores and a cache hierarchy.
} 


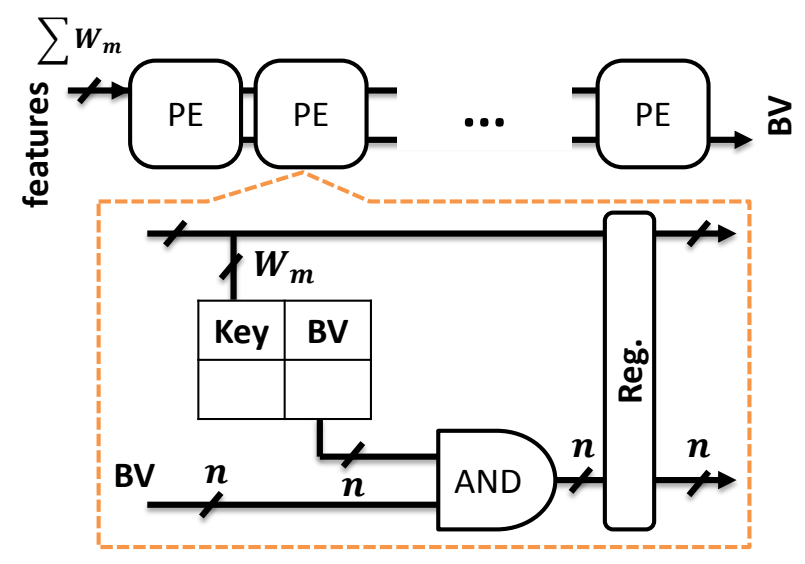

Figure 3: Architecture of a single pipeline

hash table. A BV extracted from one PE is ANDed with the BV provided from the previous PE. Hash tables are stored using dual-port distRAM or BRAM; hence the throughput is twice the maximum achievable clock rate.

\subsubsection{GPP-based Implementation}

We conducted experiments on a $2 \times$ AMD Opteron 6278 processor and a $2 \times$ Intel Xeon E5-2470 processor. The AMD processor has $162.4 \mathrm{GHz}$ cores. Each core is integrated with a $16 \mathrm{~KB} \mathrm{L1}$ data cache, $16 \mathrm{~KB}$ L1 instruction cache, and a $2 \mathrm{MB}$ L2 cache. A $6 \mathrm{MB}$ L3 cache (LLC) is shared among all the 16 cores; all the cores have access to 64 GB DDR3-1600 main memory. The AMD processor runs openSUSE 12.2 OS (64-bit 2.6.35 Linux Kernel, gcc version 4.7.1). The Intel processor has $162.3 \mathrm{GHz}$ cores. Each core has a $32 \mathrm{~KB}$ L1 data cache, $32 \mathrm{~KB} \mathrm{L1}$ instruction cache, and a $256 \mathrm{~KB}$ L2 cache. All the 16 cores share a $20 \mathrm{MB}$ L3 cache (LLC), and they have access to 48 GB DDR3-1600 main memory. This processor runs openSUSE 12.3 OS (64-bit 3.7.10 Linux Kernel, gcc version 4.7.2).

We use OpenMP on both the AMD and Intel processors. $M$ searching threads were deployed for each set of input features; they were bound to a single core in order to mitigate the penalty of data movements among the cores [14]. We used perf, a performance analysis tool in Linux, to monitor the hardware and software events such as the number of cache misses. 
Table 2: Throughput and latency of a single pipeline $(M=7)$

\begin{tabular}{|c|c|c|}
\hline No. of leaves & State-of-the-art [13] & This work \\
\hline 128 & $520 \mathrm{MCPS}, 162 \mathrm{~ns}$ & $533 \mathrm{MCPS}, 26 \mathrm{~ns}$ \\
\hline 256 & $468 \mathrm{MCPS}, 179 \mathrm{~ns}$ & $530 \mathrm{MCPS}, 26 \mathrm{~ns}$ \\
\hline 512 & $412 \mathrm{MCPS}, 204 \mathrm{~ns}$ & $523 \mathrm{MCPS}, 27 \mathrm{~ns}$ \\
\hline 1024 & $360 \mathrm{MCPS}, 233 \mathrm{~ns}$ & $437 \mathrm{MCPS}, 32 \mathrm{~ns}$ \\
\hline
\end{tabular}

\subsubsection{Trees and Data Sets}

We tested a typical decision-tree used for Internet traffic classification $[13,14]$, although our technique is not limited to decision-trees exclusively used for network applications. The imbalanced decision-tree has 42 levels, $N=92$ leaves, $M=7$ features, and classifies traffic into 8 application classes. Random traffic traces were used pessimistically ${ }^{2}$ for worst-case analysis. For synthetic decision-trees having more than 92 leaves, we used the same setup as our prior work [14].

\subsubsection{Performance Metrics}

- (Overall) Throughput: total number of classifications performed per unit time (in MCPS).

- (Processing) Latency: average processing time per classification.

\subsection{Performance on FPGA}

\subsubsection{Throughput}

We first deploy a single pipeline as shown in Figure 3 . We show the throughput of our implementation, along with the throughput achieved by the state-of-the-art implementation in Table 2. As can be seen, we achieve better throughput since the wire length of our pipelined architecture is reduced compared to the state-of-the-art implementation.

The resource consumption shown in post-place-and-route reports indicates that less than $30 \%$ of the total resources (logic slices and I/O pins) are used for a single pipeline. Hence we deploy 3 pipelines to improve the throughput of our classification engine; we show in Figure 4 the throughput with respect to various values of $N$ and $M$. Large values of $N$ deteriorate

\footnotetext{
${ }^{2}$ Low cache efficiency for our implementations on multi-core GPP.
} 


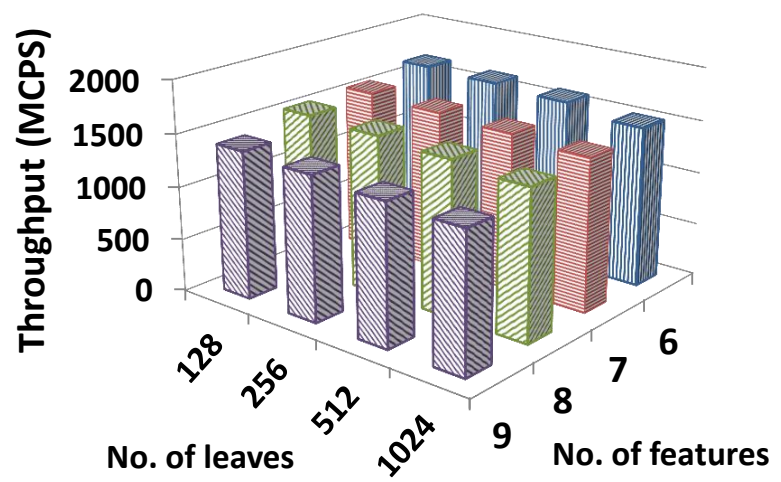

Figure 4: Varying $N$ (depleting FPGA resources using 3 pipelines)

the clock rate of each $\mathrm{PE}^{3}$; large values of $M$ deteriorate the throughput ${ }^{4}$ and prolong the pipeline latency (see Section 4.2.2). However, as can be seen in Figure 4, the throughput loss is less than $8 \%$.

\subsubsection{Latency}

For multiple pipelines deployed in parallel, the processing latency is the same as the single-pipeline latency. We show in Table 2 the worst-case latency performance. As can be seen, the state-of-the-art implementation incurs long latency (42 pipeline stages). However, our pipelined architecture only uses a small number of pipeline stages while sustaining high clock rate. Note we have a speedup of around $6 \sim 7 \times$ for the latency performance.

\subsection{Performance on Multi-core GPP}

On multi-core GPP, a large number (denoted as $P$ ) of concurrent packet classifiers can be deployed; this is different from our designs on FPGA, where only a small number of pipelines can be employed. We choose $P=32$ based on our prior work [14]. In addition, thanks to the memory hierarchy on multi-core GPP, larger decision-trees and more features can be supported.

We show in Figure 5 the throughput and latency performance on our $\mathrm{AMD}$ and Intel platforms, with respect to various values of $M$ and $N$. As $M$ or $N$ increases, the performance tapers; the reasons are:

\footnotetext{
${ }^{3}$ This is because large hash tables are required in each PE for large $N$.

${ }^{4}$ More resources are used, leading to less place-and-route choices and inferior clock rate.
} 

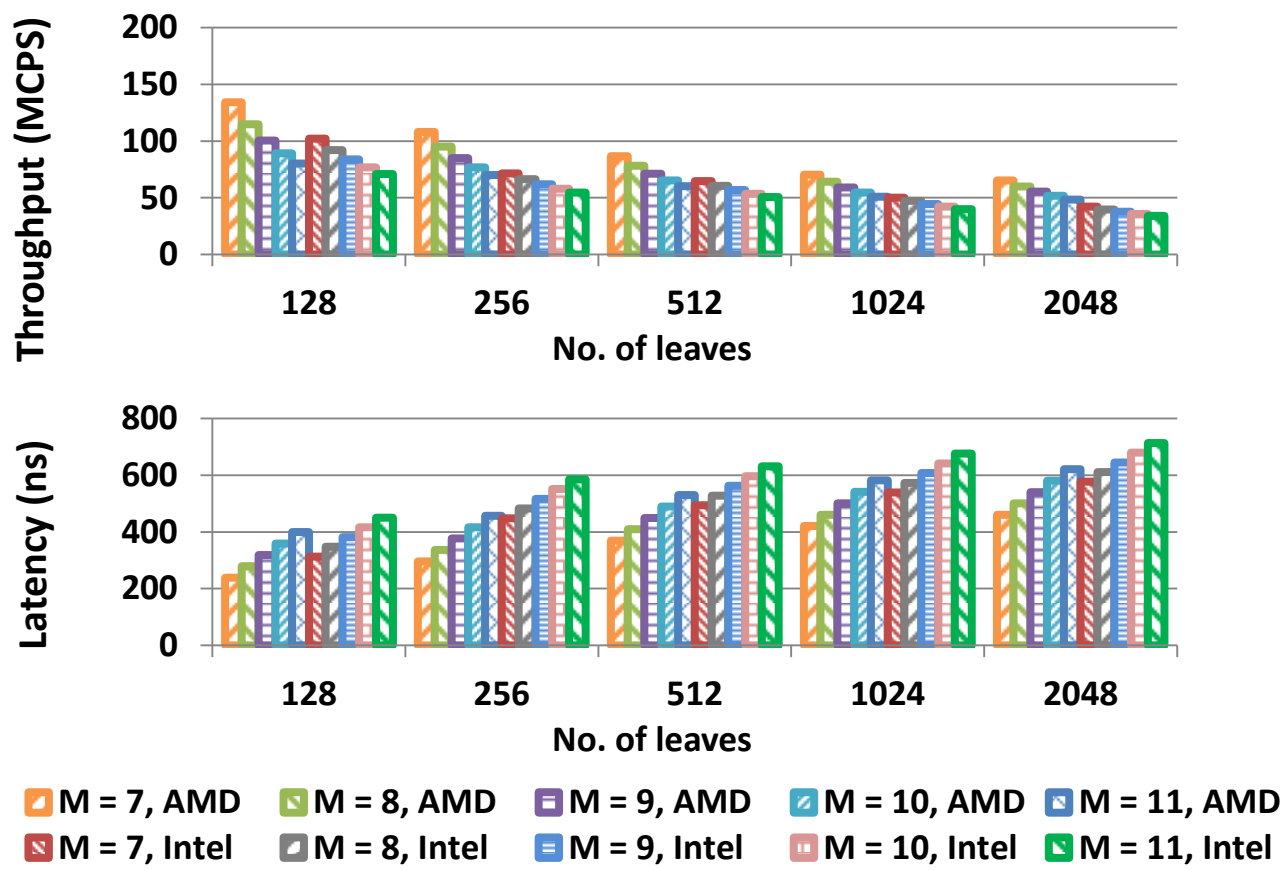

Figure 5: Performance on multi-core GPP ( $P=32$ concurrent classifiers)

1. More memory accesses are required for larger values of $M$ since more hash tables need to be examined.

2. Cache performance degrades for large values of $N$. Given that the number of key-BV pairs $q^{(m)}$ is kept proportional to $N$, it is more difficult to fit larger hash tables in cache for larger $N$.

A detailed analysis [14] reveals that the searching time contributes $80 \% \sim$ $90 \%$ of the processing latency. The AMD platform achieves better performance than the Intel platform; note the AMD processor has a higher clock frequency. Compared to the Intel platform, the AMD processor misses less in L2 cache but more in L3 cache [14], due to their different cache sizes; this can also result in better performance on the AMD platform. For $M=7$, $N=92$, and $P=32$, we achieve 134 MCPS throughput and 239 ns latency on the AMD platform, and 102 MCPS throughput and 313 ns latency on the Intel platform, respectively. 


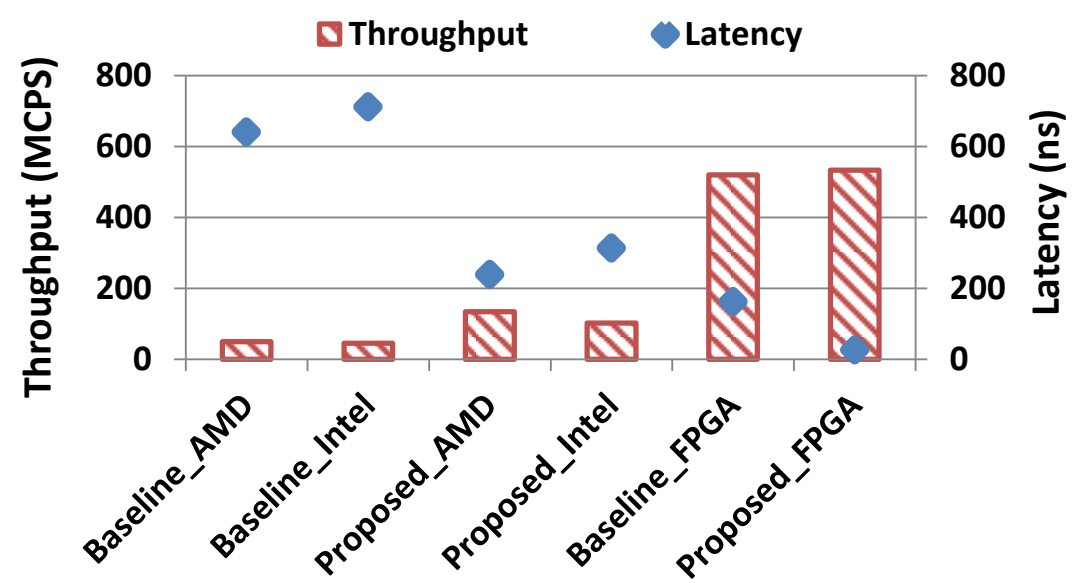

Figure 6: Performance comparison

\subsection{Comparison}

As an example, we study a typical C4.5 decision-tree consisting of 42 levels, 92 leaves, and 7 features. In Figure6, we show the throughput and latency performance for various implementations on software-based and hardware-based platforms. As baselines on multi-core GPPs, we denote the straightforward Binary Search Tree (BST) implementations on the AMD and Intel platforms as "Baseline_AMD" and "Baseline_Intel", respectively; our implementations based on compact hash tables are denoted as "Proposed_AMD" and "Proposed_Intel", respectively. Similarly, the state-ofthe-art implementation of the decision-tree on FPGA is remapped onto our Virtex-7 FPGA, and denoted as "Baseline_FPGA"; our implementation based on compact hash tables are denoted as "Proposed_FPGA". As can be seen:

- The FPGA-based approaches achieve better performance; this is because the application-specific architectures on FPGA are designed and optimized with parallel accesses to SRAM. However, multi-core GPPs can support much larger decision-trees (larger $N$ ) or a larger number of features (larger $M$ ) than FPGA, due to its memory hierarchy.

- On both the multi-core GPP and FPGA platforms, our proposed approaches using compact hash tables achieve better performance $(2.7 \times$ and $6 \times$ speedups, respectively). 


\section{Conclusion}

In this paper, we presented a translation from a decision-tree to multiple compact hash tables. We evaluated and compared the performance on stateof-the-art platforms. Our future work includes exploring efficient merging of searching results and investigating the performance on GPU-based platforms.

\section{References}

1. Sedgewick R. Left-Leaning Red-Black Tree. 2008. URL http://www.cs.princeton.edu/ rs/talks/ LLRB/LLRB. pdf.

2. Cormen TH, Leiserson CE, Rivest RL, Stein C. Introduction to Algorithms. 3 ed.; The MIT Press; 2009.

3. Virtex-7 FPGA Family. 2012. URL http://www.xilinx.com/products/silicon-devices/fpga/ virtex-7/index.htm.

4. Hellerstein JM, Naughton JF, Pfeffer A. Generalized Search Trees for Database Systems. In: Proc. of the 21th Intl. Conf. on Very Large Data Bases (VLDB). 1995:562-573.

5. Sheldon D, Vahid F. Don't Forget Memories: A Case Study Redesigning a Pattern Counting ASIC Circuit for FPGAs. In: Proc. of IEEE/ACM/IFIP Intl. Conf. on Hardware/software Codesign and Sys. Syn. (CODE+ISSS). 2008:155-160.

6. Chandramowlishwaran A, Madduri K, Vuduc R. Diagnosis, Tuning, and Redesign for Multicore Performance: A Case Study of the Fast Multipole Method. In: Proc. ACM/IEEE High Perf. Comp., Netw., Storage and Analysis (SC). 2010:1-12.

7. Ma Y, Banerjee S, Lu S, Estan C. Leveraging Parallelism for Multi-dimensional Packet Classification on Software Routers. SIGMETRICS Perform Eval Rev 2010;38(1):227-238.

8. Pilla L, Ribeiro C, Cordeiro D, Mei C, Bhatele A, Navaux P, Broquedis F, Mehaut J, Kale L. A Hierarchical Approach for Load Balancing on Parallel Multi-core Systems. In: Proc. of Intl. Conf. Parallel Processing (ICPP). 2012:118-127.

9. Bader D, Kanade V, Madduri K. SWARM: A Parallel Programming Framework for Multicore Processors. In: Proc. of IEEE Intl. Para. Dist. Proc. Symp. (IPDPS). 2007:1-8.

10. Sarje A, Aluru S. Parallel Applications Employing Pairwise Computations on Emerging Architectures. In: Proc. of IEEE Intl. Para. Dist. Proc. Symp. Workshop (IPDPSW). 2010:1-4.

11. Ali S, Eslamnour B, Shah Z. A Case for On-machine Load Balancing. J Parallel Distrib Comput 2011;71(4):556-564.

12. Liang C, Zhang Y, Song Q. Decision Tree for Dynamic and Uncertain Data Streams. In: Proc. of ACML. 2010:209-224.

13. Tong D, Sun L, Matam K, Prasanna V. High Throughput and Programmable Online Trafficclassifier on FPGA. In: Proc. of ACM/SIGDA Intl. Symp. on Field Prog. Gate Arrays (FPGA). 2013:255264

14. Qu Y, Prasanna V. Compact Hash Tables for High-Performance Traffic Classification on Multi-core Processors. In: Proc. of IEEE Intl. Symp. on Comp. Arch. and High Perf. Comp. (SBAC-PAD). 2014:17-24.

15. Pagh R, Rodler FF. Cuckoo Hashing. Springer; 2001. 